\title{
Focal transformation, an imaging concept for signal restoration and noise removal
}

\author{
A. J. Berkhout ${ }^{1}$ and D. J. Verschuur ${ }^{2}$
}

\begin{abstract}
Interpolation of data beyond aliasing limits and removal of noise that occurs within the seismic bandwidth are still important problems in seismic processing. The focal transform is introduced as a promising tool in data interpolation and noise removal, allowing the incorporation of macroinformation about the involved wavefields. From a physical point of view, the principal action of the forward focal operator is removing the spatial phase of the signal content from the input data, and the inverse focal operator restores what the forward operator has removed. The strength of the method is that in the transformed domain, the focused signals at the focal area can be separated from the dispersed noise away from the focal area. Applications of particular interest in preprocessing are interpolation of missing offsets and reconstruction of signal beyond aliasing. The latter can be seen as the removal of aliasing noise.
\end{abstract}

\section{INTRODUCTION}

In the history of temporal and spatial seismic processing two issues have always played a central role: signal-to-noise ratio and resolution. Noise removal methods are based on the principle that a suitable transformation brings the data into a new domain where signal and noise can be well separated. Very familiar are the Fourier transform and the Radon transform. More recently, multidimensional wavelet transforms have received ample attention, allowing the differentiation of signal from noise by different local-scale behavior (Candès and Guo, 2002; Zhang and Ulrych, 2003; Herrmann and Moghaddam, 2004).

The most important aspect of noise removal is the design of a suitable transformation. In this paper, we argue that an effective and ro- bust method is obtained if the transformation contains macroinformation about physical properties that underlie the measurement. For seismic processing, this means that wave-propagation properties should be used in the transformation. The idea of using wave properties in the transformation has been exploited by several authors in the past. Harlan et al. (1984) discussed focusing methods to exploit the local coherence of the signal and to make the noise more Gaussian. Recently, Trad (2003) introduced a migration-demigration procedure to interpolate seismic data. In the following, the focal transform is introduced. This transform is based on a multidimensional operator that contains an estimate of the propagation properties in the subsurface. Implementation occurs by normalized crosscorrelation as used in the second step of bifocal migration (Berkhout, 1997). This means that the focal transform represents a multishift correlation process that maps data into and around one focal point. The multishift property makes the focal transform fundamentally different from Fourier and Radon-like methods, as well as migration-demigration concepts. It means that the inverse focal transformation is well defined by a multishift convolution process (defocusing). We will see that the focal domain offers new opportunities in interpolation and separation.

\section{PRINCIPLE OF THE FOCAL TRANSFORM}

Let matrix $\mathbf{P}$ represent any prestack 3D data volume, one column representing one monochromatic shot record, and let matrix $\mathbf{G}$ represent a suitable 3D propagation operator (Berkhout, 1982). Then we will formulate forward and inverse focal transformation as a matrix multiplication per temporal frequency component:

$$
\mathbf{Q}=\mathbf{F P} \text { (forward) }
$$

and

$$
\mathbf{P}=\mathbf{G Q} \text { (inverse) }
$$

Manuscript received by the Editor March 1, 2006; revised manuscript received June 3, 2006; published online October $26,2006$.

${ }^{1}$ Delft University of Technology, Faculty of Technology, Policy and Management, P.O. Box 5015, 2600 Ga Delft, the Netherlands. E-mail: a.j.berkhout@ tudelft.nl.

${ }^{2}$ Delft University of Technology, Faculty of Applied Sciences, Laboratory of Acoustical Imaging and Sound Control, P.O. Box 5046, 2600 Ga Delft/Lorentzweg 1,2628 CJ, Delft, the Netherlands.E-mail: d.j.verschuur@tudelft.nl.

(C) 2006 Society of Exploration Geophysicists. All rights reserved. 
with

$$
\mathbf{F}=\mathbf{G}^{-1}
$$

If we use a weighted least-squares inversion approach, the forward focal operator can be written as
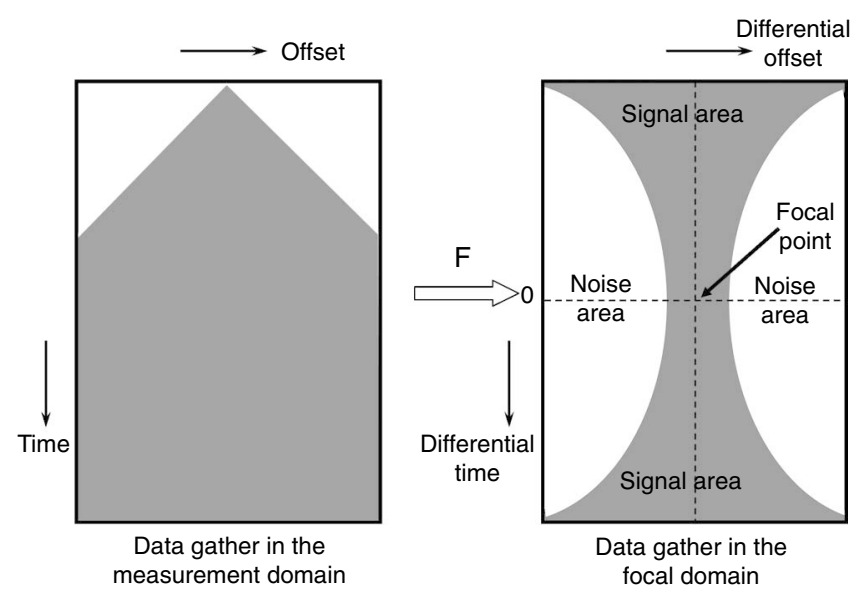

Figure 1. The focal transform localizes the nonaliased signal energy around its focus point and disperses the aliased signal and coherent noise away from its focal area. Here, a schematic example of a signal window is shown.

$$
\begin{aligned}
\mathbf{F} & \approx \mathbf{G}^{H}\left[\mathbf{G G}^{H}+\varepsilon^{2} \mathbf{I}\right]^{-1} \quad \text { (least squares) } \\
& =\mathbf{G}^{H} \mathbf{B},
\end{aligned}
$$

with

$$
\mathbf{B}=\left[\mathbf{G G}^{H}+\varepsilon^{2} \mathbf{I}\right]^{-1}
$$

and

$$
\mathbf{Q}=\mathbf{G}^{H} \mathbf{B} \mathbf{P} \quad \text { (weighted correlation), }
$$

$\varepsilon$ being a small stabilization constant.

From a physical point of view, correlation operator $\mathbf{G}^{H}$ removes spatial phase from a recording and weighting operator $\mathbf{B}$ inverts the amplitude spectrum of $\mathbf{G}$ in a stabilized manner. Convolution operator $\mathbf{G}$ restores what $\mathbf{F}$ has removed. Note that the phase spectra of $\mathbf{F}$ and $\mathbf{G}$ are always opposite and thus have no influence on the output. This property makes the focal transform very robust.

For a suitable choice of $\mathbf{G}$, focal transformation reduces the spatial phase of reflections, causing the reflection energy to become more localized. In the extreme case that $\mathbf{G}$ equals the reflection wavefield in a recording, focal transformation means weighted autocorrelation. As a result, the spatial phase will be zero and the spatial amplitude will be close to unity. More specifically, the reflection energy of a common-midpoint (CMP) gather will be localized around its midpoint, the reflection energy of a shot record will be localized around its shotpoint, and the reflection energy of a receiver gather will be localized around its receiver point. This unique property provides the opportunity to separate the focused signal from the dispersed noise more effectively than existing methods (see Figure 1). In addition, by inverse transformation any desired sampling distribution of the output may be chosen.

In Figure 2 we illustrate the principle on a single reflector case with some added background noise (Figure 2a). The operator is shown in Figure $2 \mathrm{~b}$, which is chosen to be identical to the reflection event in Figure 2a. Forward focal transformation transforms the reflector response toward the source point at and around the origin (Figure 2c). This can be explained by realizing that the correlation property of the focal transform reduces the spatial phase in the data. The smaller the residual phase, the more the signal energy will be localized in the focal domain. In this illustration, the residual phase is zero. Note that the noise does not match the phase of the operator. It is therefore distributed all over the focal data space. In this data space, filtering should occur by using the property that the desired signal energy can be found around the origin (the focus point is a bandlimited spike). Note that in the linear Radontransformed focal data space, the focused signal can be found at and around $\tau=0$. It is important to realize that, without filtering, inverse transformation retrieves the original data within the limitation of the stabilized least-squares inversion (Figure 2d). This is also true if the operator leaves some residual phase in the focal data space. The only requirement is that $\mathbf{F}=\mathbf{G}^{-1}$. In Figure $2 \mathrm{e}$ and $f$, the result of forward and inverse focal transformation is shown for an operator that does 
not match the reflector response because it contains a velocity error of $2 \%$. The residual phase is clearly visible in the focal domain; compare Figure $2 \mathrm{c}$ and e. It has, however, no influence on the output; compare Figure $2 \mathrm{~d}$ and $\mathrm{f}$.

Bear in mind that for this example, the earth is laterally invariant, meaning that all shot records are identical and that the data matrix $\mathbf{P}$ and the propagation operator $\mathbf{G}$ in equation 1 exhibit a Toeplitz structure. Then, the calculation of the focal operator $\mathbf{F}$ in equation 4 can be carried out for a single gather by scalar operations in the horizontal wavenumber-frequency domain. However, if the earth is laterally inhomogeneous, all operations must be carried out as matrix operations in the space-frequency domain.

It is also important to realize that in the focal transform, operators can be single reflector or multireflector. In this paper, we will focus on multireflector operators. This means that operators represent simulated or preprocessed data gathers, containing primaries only or primaries and multiples. The multireflector focal transformation concept deviates fundamentally from current methods and, therefore, it may lead to new opportunities in seismic processing.

\section{MULTIREFLECTOR FOCAL OPERATORS}

If $\mathbf{G}(m)$ represents a single-reflector operator, then single-reflector operators can be combined into one multireflector operator gather:

$$
\mathbf{G}=\sum_{m} \mathbf{G}(m)
$$

$m$ indicating some time level.

This means that the focal transform is applied to all these time levels at the same time. The operator gather may be the output of a preprocessing step (data-driven version) or it may be the output of a modeling step (model-driven version). Application of multireflector focal operator $\mathbf{F}=\mathbf{G}^{-1}$ to the related data gather transforms all reflection energy of the gather toward the focal point. For a commonsource gather (shot record), this means that all energy is propagated back toward its source point; for a common-detector gather, all energy is propagated back toward its detector point; and for a commonmidpoint gather, all energy is propagated back toward its midpoint.

In Figure 3, we illustrate the multireflector focal transform on a 13-reflector CMP gather ( $\Delta x=15 \mathrm{~m})$, using only the five main reflectors in the operator $(\Delta x=15 \mathrm{~m})$. The events in the operator are modeled by picking the five strongest points in the velocity semblance panel, after which for each pick a hyperbolic event has been created with amplitudes that honor geometric spreading in a homogeneous medium. Note that the focal area around the origin contains the focused energy of all reflections. Outside the focal point, we find the sum of all cross products. They cancel in the situation of a perfect operator. After inverse transformation, the input data are retrieved (Figure 3d), despite the imperfect operator. As mentioned earlier, this is an important property of the focal transform.

Next, every other trace is removed from the input data set, yielding an aliased CMP gather with $\Delta x=30 \mathrm{~m}$ (Figure 4a). With the multireflector operator (Figure 3b), the focal transformation is applied (see Figure 4b). Note that the aliased energy maps into the area as indicated in Figure 1. This energy can be removed by windowing (Figure 4c). Finally, the windowed focal domain data is inverse transformed according to equation 2 . The newly obtained traces are merged with the original traces, yielding an interpolated CMP gather
(Figure 4d). The Fourier spectrum of input and output in Figure 5 shows that the aliasing energy has been well removed.

In the case of multireflector operators, the focal point contains information about the multiangle strength of the involved sources and the multiangle sensitivity of the involved detectors. For a shot record, this multiangle information refers to the involved source strength, and for a detector gather, this multiangle information refers to the involved detector sensitivity. We currently investigate this property for angle-dependent equalization purposes in the Radontransformed focal domain.

If the input consists of both primaries and multiples, and we use in the focal transform an operator $\mathbf{G}$ that contains the primary reflections only, i.e., $\mathbf{G}=\Delta \mathbf{P}$, then $\mathbf{F}=\Delta \mathbf{P}^{-1}$ and all primary reflections are mapped onto the origin (focal point) and all multiples will appear at positive times. This property of the focal transform opens a new possibility to separate primaries and multiples in the focal domain (Berkhout and Verschuur, 2006).

If we use in the focal transform an operator $\mathbf{G}$ that contains the seismic data with multiple scattering, i.e., $\mathbf{G}=\mathbf{P}$, then the focal operator $\mathbf{F}$ represents the seismic data in the inverse data space (Berkhout, 2006):

$$
\mathbf{F}=\mathbf{P}^{-1}=\mathbf{P}_{0}^{-1}-\mathbf{A},
$$

$\mathbf{P}_{0}$ representing the data without surface-related multiples and $\mathbf{A}$ representing the source and detector information. a)
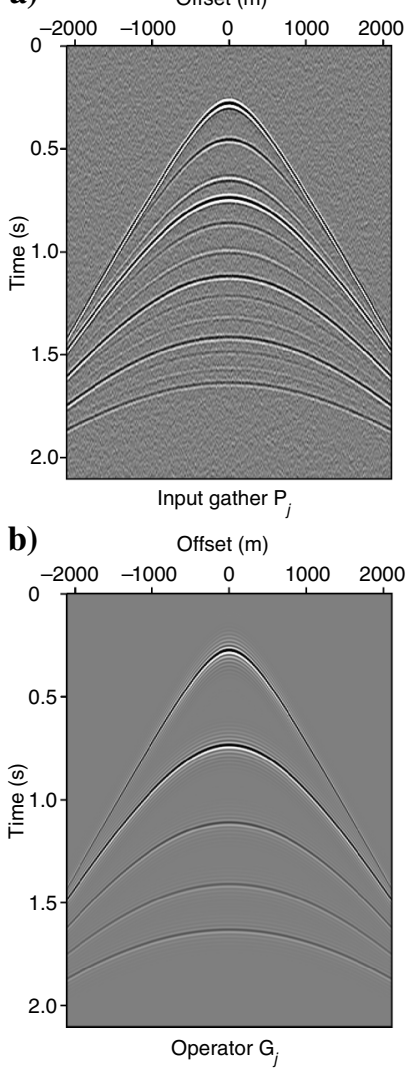

c) Offset $(\mathrm{m})$

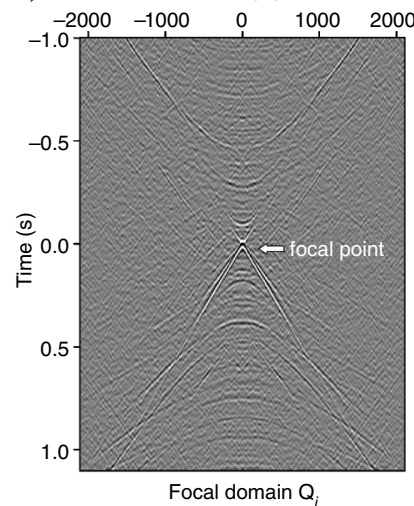

d)

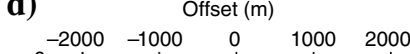

Figure 3. Input CMP gather with 13 reflections (a) and a multireflector operator containing five events only (b). Focal transformation of the data in (a) with focal operator (b) yields the focal domain (c). Inverse transformation of (c) yields the output gather (d). Note again that without filtering in the focal domain, the signal output equals the signal input. Note also that the large amplitude at the focal point has been clipped for display purposes. 
a)

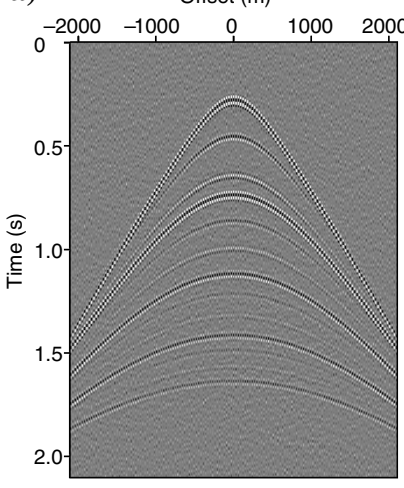

Input gather $\Delta \mathrm{x}=30 \mathrm{~m}$

b)

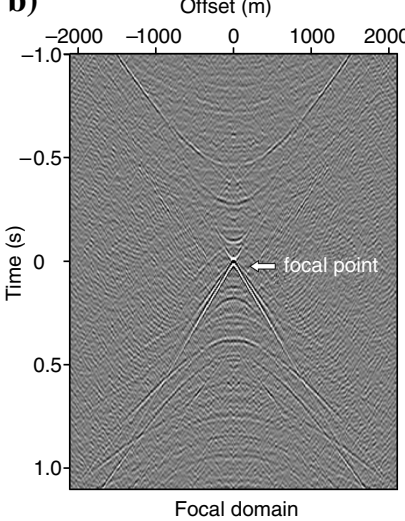

c)

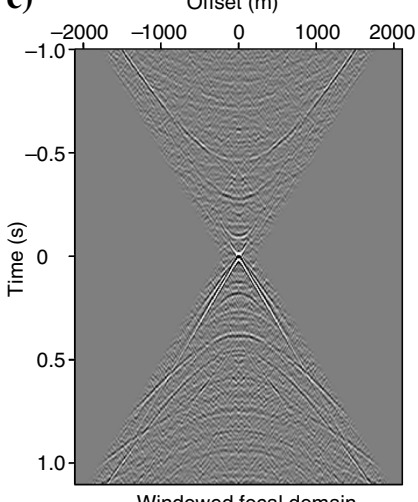

d)

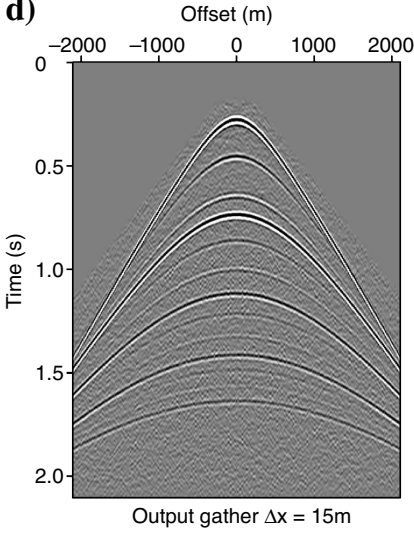

Figure 4. Focal transformation applied to a 13-reflector input gather with 30-m trace spacing (a) using a five-reflector operator with $15-\mathrm{m}$ trace spacing (Figure $3 \mathrm{~b}$ ). The energy of the reflections is mapped at and around the focal point, whereas aliasing noise is mapped on the left- and right-hand side (b). After removing the aliased noise in the focal domain by windowing (c) and inverse transformation, the interpolated traces are inserted in the input data $(\mathrm{d})$.

\section{COMPARISON WITH RADON TRANSFORMATION AND MIGRATION-DEMIGRATION}

Comparing our focal transform concept with the well-known family of Radon transformation and migration-demigration methods, we can state the following:

- Focal transformation does not use a single-shift operation, as we see in the Radon and migration process. It applies a multishift algorithm.

- Focal transformation does not apply any imaging principle, meaning that full reconstruction is possible.

- Focal transformation, as proposed by us, does not use a single event operator. It applies a multireflector operator gather.

In addition, the focal transform concept can also be related to diffraction events, meaning that the focal operator contains macroinformation of the diffractions in the data. Finally, note that the focal transform concept can also be related to noise events, meaning that the focal operator contains phase information of the noise in the data.

a) Hor. Wavenumber $(1 / \mathrm{m})$

b) Hor. Wavenumber $(1 / \mathrm{m})$
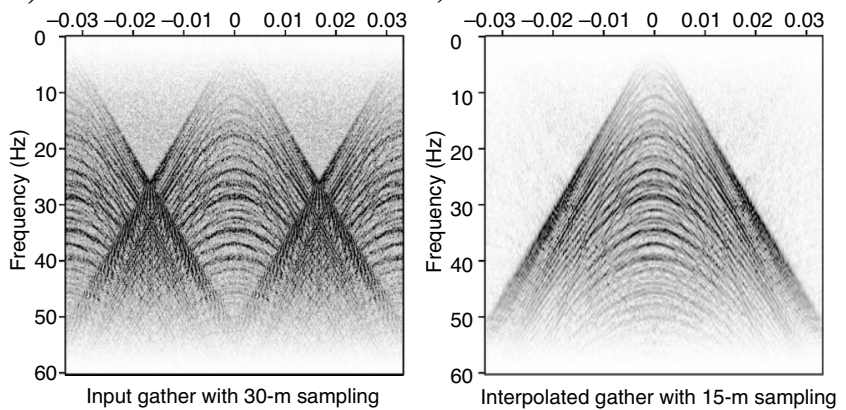

Figure 5. (a) The $f-k$ spectrum of the aliased input gather of Figure 4a. (b) The $f-k$ spectrum of the interpolated gather of Figure $4 \mathrm{~d}$. Note the effective suppression of the aliasing noise.

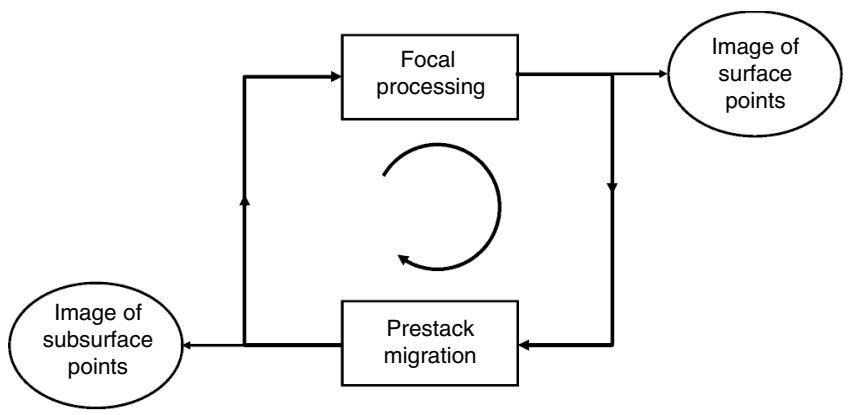

Figure 6. Dual imaging strategy: imaging of surface points is used to recover missing data and estimate source/detector properties; imaging of subsurface points is used to obtain propagation operators and reflection properties. The double imaging process is supposed to be carried out in a recursive manner, aiming at images of both the surface (acquisition points) and subsurface (reflection points).

\section{CONCLUSIONS}

Using macroinformation of wavefields, multireflector focal operators can be constructed. These operators are used to image surface points - source/detector/mid — followed by removing signal-related noise away from the imaged surface points (focal points). The output data can be used for a next iteration.

The focal transform concept is fundamentally different from Fourier- and Radon-like algorithms as well as the family of migrationdemigration methods. Single operators are replaced by operator gathers, and dot products are replaced by convolutions or deconvolutions. The concept of operator gathers is broad. Such a gather may contain primary scattering and/or multiple scattering, or linear noise.

Extrapolating from the presented theory, we expect that focal transformation, i.e., imaging surface points, in combination with seismic migration, i.e., imaging subsurface points, will introduce new opportunities in seismic imaging (see Figure 6).

\section{ACKNOWLEDGMENTS}

The authors thank the sponsors of the Delphi consortium for their assistance, Remco Romijn for his assistance in generating the examples, and Mauricio Sacchi for the helpful discussions during his sabbatical year at Delft University. 


\section{REFERENCES}

Berkhout, A. J., 1982, Seismic migration, imaging of acoustic energy by wave field extrapolation, A: Theoretical aspects, 2nd ed.: Elsevier Science Publishing Co., Inc.

-, Pushing the limits of seismic imaging, Part II: Integration of prestack migration, velocity estimation and AVO analysis: Geophysics, 62, 954969.

, Seismic processing in the inverse data space: Geophysics, 71, A29A33.

Berkhout, A. J., and D. J. Verschuur, 2006, Imaging of multiple reflections: Geophysics, 71, SI209-SI220.
Candès, E. J., and F. Guo, 2002, New multiscale transforms, minimum total variation synthesis: Applications to edge-preserving image reconstruction: Signal Processing, 82, 1519-1543.

Harlan, W. S., J. F. Claerbout, and F. Rocca, 1984, Signal/noise separation and velocity estimation: Geophysics, 49, 1169-1180.

Herrmann, F. J., and P. P. Moghaddam, 2004, Curvelet-based nonlinear adaptive subtraction with sparseness constraints: 74th Annual International Meeting, SEG, Expanded Abstracts, 1977-1980.

Trad, D. O., 2003, Interpolation and multiple attenuation with migration operators: Geophysics, 68, 2043-2054.

Zhang, R., and T. Ulrych, 2003, Physical wavelet frame denoising: Geophysics, 68, 225-231. 